\title{
Phytochemical Screening of Adathodai Kudineer A Siddha Herbal concoction and Evaluation of its binding affinity with SARS-CoV-2 Spike Protein and ACE2 Receptor Spike protein Complex through Molecular Docking in silico approach
}

\author{
Research Article
}

\section{Christian G J1*, Meenakumari R², Rajamaheswari K², Priyanka Sekaran ${ }^{4}$, Gajalakshmi G5, Anand T6}

1. Professor, 2. Director, 3. Ph.D Scholar, 4. PG Scholar, 5. PG Scholar, National Institute of Siddha, Chennai. 6. Assistant Professor, Department of Genetic Engineering, SRM Institute of Science and Technology, Chennai.

\begin{abstract}
Adathodai kudineer (AK) a Classical Siddha formulation is used to treat various fevers which cause moderate to severe acute respiratory symptoms as is indicated in the text. GC-MS analysis was carried out to identify the presence of potent lead molecules in $A K$ against novel corona virus. The aqueous extract has shown the following bioactive compounds such as Napthalene, Benzene Propanol, Benzene Acetic Acid, Furazan-3-amine, Pyrazol-4Carboxylic acid, 2(3H) Furanone. The ethanolic extract of $A K$ exhibited the molecular compounds such as Eucalyptol, Toluene, 2-Carene, Alpha-Copaene, 1,6-Cyclodecadiene, Aromadendrene, Gamma-murolene, Betacopaene, Cubebol, Selina-3,7 (11) - Diene, 2-Butanone. Molecular docking is a powerful approach in current trends to identify the possibility of pharmacological effects of medicinal compounds which could be exerted over their Corresponding Protein targets which are relevant for causing disease. Using Auto dock Vina Software, the biomolecules of $A K$ were analyzed through molecular docking against SARS-CoV-2 Spike Protein (PDB ID 6LU7) and SARS-CoV-2 Spike Protein - ACE 2 receptor complex (6LZG). ADME properties were also recorded for the aqueous and ethanolic extracts of $A K$ compounds using online tool SWISS ADME. The binding energy observed were of the order: $-10.9 \mathrm{Kcal} / \mathrm{mol},-8.0 \mathrm{Kcal} / \mathrm{mol},-7.8 \mathrm{Kcal} / \mathrm{mol}$ for the compounds Alpha-Copaene, GammaMurolene, Selina-3,7 (11)-Diene respectively towards the protein target $6 \mathrm{LZG}$ and $-8.2 \mathrm{Kcal} / \mathrm{mol}$, $-6.6 \mathrm{Kcal} / \mathrm{Mol}$, $-6.5 \mathrm{Kcal} / \mathrm{Mol}$, for the compounds Alpha-Copaene, Cubebol, Aromadendrene respectively towards for the target 6LU7. These findings confirm that the Siddha formulation Adathodai Kudineer has some potent activity against SARS-CoV-2 Virus COVID19 disease.
\end{abstract}

Key Words: Adathodai kudineer, ADME, Siddha medicine, Protein-Protein Interaction, Molecular Docking, COVID 19.

\section{Introduction}

Emerging of Viral diseases like SARS, H1N1 influenza, MERS-CoV as epidemic in past decades. Now the current scenario in the entire world is facing a pandemic situation of Corona Viral infection which first emerges in Wuhan, China. World health organization declared the global health emergency due to COVID19 outbreak. The COVID 19, a disaster of 2020 has affected almost all the countries of the world with increasing death rates. As of 24 April 2021, there have been 145,216,414 confirmed cases of COVID-19, including 3,079,390 deaths, reported to WHO (1). It was identified that the Novel Beta Corona Virus named SARS$\mathrm{CoV} 2$ is the causative organism behind the outbreak of COVD19.

* Corresponding Author:

\section{Christian G J}

Professor,

National Institute of Siddha,

Chennai,

Tamilnadu. India.

Email Id: christianvijila@gmail.com
It is currently estimated that the incubation of SARS-CoV2 infection is between 2-14 days before the symptom's onset such as fever, cough, Sore Throat, dyspnea and the median disease incubation period was calculated as 5.1 days by the researchers of Johns Hopkins Bloomberg School of Public Health. The rapid transmission is occurred in COVID 19 is due to its person to person transmission is highly efficient and through respiratory droplets. The SARS-CoV2 is infecting the host cells by entering into the Human ACE 2 receptors through its enveloped spike glycoprotein (2). The primary target of the COVID 19 causing virus is the lung epithelial cells. The risk factors were assessed based on the available clinical data such as Hypertension, Cardiovascular Conditions, Cancer and Diabetes Mellitus.

Research Studies are being conducted to identify the impairment in response of antibody to SarsCoV2 infection, quantification of viral load, viral shedding prolongment in immunocompromised COVID 19 affected individuals. Immunity plays a significant role while challenging with viral infections. Some traditional Siddha medicines are being unique in treating viral infections at various stages including at the time of pathogenesis, prognosis due to its potent antiviral pharmacological 
effects. Potent antiviral Siddha drug Nilavembu kudineer was documented as a wonderful therapeutic drug for the treatment of Chikungunya and dengue viral fever in both preclinical as well as clinical aspects(3)(4)(5). Likewise, identification of an antiviral drug with high therapeutic efficacy against COVID 19 infection is the urgent need in the current scenario because the medical world is only challenging and facing the disease using existing antiviral drugs which was identified for various other viral infections not exactly for SARS-CoV2.

Siddha system of medicines is a boon to human kind in treating various kind of infectious fever. According to Siddha pathology the disease COVID 19 is caused due to the elevation of Kapha humour while correlating its signs and symptoms. Adathodai Kudineer is a sastric Siddha medicine consists of various medicinal herbs was indicated to treat various fevers. Adathodai kudineer has been studied widely for its Anti pyretic and the individual ingredients seems to have multiple effects including AntiBacterial, Anti-fungal, Anti-Viral, Immuno Modulatory and much more (6). Since there is no proper drug was identified for the treatment of Corona Viral disease 19, the Siddha formulation Adathodai Kudineer have to be investigated for its Anti-corona Viral activity. Molecular docking analysis is an easiest and an effective way to identify the action potential of biomolecules of a drug against disease causing viral protein. GCMS was used to identify the presence of bioactive ingredients in Siddha drug Adathodai Kudineer and the minimum binding affinity of those biomolecules of KSK was found against Corona virus spike protein molecule and its ACE2 binding domain complex through molecular docking analysis. ADME properties was also evaluated for those compounds.

\section{Materials and Methods}

\section{Source of drug}

The Sastric drug Adathodai Kudineer was prepared as per the Siddha text Siddha Vaithya Thirattu (7) (8). The drug AK has the following 4 herbals such as Adathodai (Justicia adhatoda L.), Adhimadhuram (Glycyrrhiza glabra L.), Thaalisapathiri (Taxus buccata L.), Arisi Thippili (Piper longum L.).

\section{GCMS analysis}

GCMS analysis was carried out on the Aqueous and Ethanolic extract of Siddha drug Kabasura kudineer at the NABL accredited laboratory (Bureau veritas) in Chennai as per the standard protocol.

\section{Protein preparation}

TheSARS-CoV-2Virus main protease complex with an inhibitor N3 3D structure (PDBid: 6LU7) and Spike protein bound with ACE2 receptor protein molecule structure (PDBid:6LZG) was retrieved from the Protein Data Bank (PDB) and energy minimized and then converted into their respective PDBQT formats.2d. Ligand preparation:

The lead molecules were identified in the Siddha formulation achieved through GCMS analysis such as Napthalene, Benzene Propanol, Benzene Acetic Acid, Furazan-3-amine, Pyrazol-4-Carboxylic acid, 2(3H) Furanone, Eucalyptol, Toluene, 2-Carene, Alpha-Copaene, 1,6-cyclodecadiene, Aromadendrene, Gamma-murolene, Beta-copaene, Cubebol, Selina-3,7 (11) - Diene, 2Butanone were selected as ligands for docking. 3D structures of the above mentioned selected ligands taken from PubChem structures and were minimized by applying Gnagstiere charge and coleman charge the root of the structure was detected torsion applied and the structures were saved in PDBQT format which is regularly used in Molecular docking analysis (9).

\section{Molecular Docking}

Molecular Docking analysis performed with a commonly well-known established software Auto dock Vina a very convenient and excellent screening tool for identifying binding energy between the 3D structures of each ligand and target proteins (10). Two target proteins PDB ID: 6LU7 and PDB ID: 6LZG were selected, a Gridfree docking performed, and the binding energies of each ligand found.

\section{ADME properties prediction}

The ADME (Adsorption, dissociation, Metabolism and excretion properties of above mentioned natural compounds predicted using Swiss ADME an online tool for ADME prediction (11).

\section{Protein-Ligand interaction profile}

Amino acids interactions of the ligands and their relation with the natural bonds were identified using a software tool Ligplot++(10). All the results were summarized and interpreted.

\section{Results}

Table 1. Phytocomponents Identified In The Aqueous Extract Of Medicinal Compound AdathodaiKudineer

\begin{tabular}{|c|c|c|c|c|c|}
\hline S.No & RT & Area \% & $\begin{array}{c}\text { Library/ID } \\
\text { (CDDatabaselNIST11.L) }\end{array}$ & Mol. F & Mol. W (g/mol) \\
\hline 1 & 12.430 & 24.69 & Naphthalene & $\mathrm{C}_{10} \mathrm{H}_{8}$ & $128.169 \mathrm{~g} / \mathrm{mol}$ \\
\hline 2 & 12.989 & 18.34 & Benzene Propanol & $\mathrm{C}_{9} \mathrm{H}_{12} \mathrm{O}$ & $136.19 \mathrm{~g} / \mathrm{mol}$ \\
\hline 3 & 12.989 & 18.34 & Benzene Acetic Acid & $\mathrm{C}_{9} \mathrm{H}_{10} \mathrm{BrNO}_{2}$ & $244.08 \mathrm{~g} / \mathrm{mol}$ \\
\hline 4 & 24.257 & 13.93 & Furazan-3-Amine & $\mathrm{C}_{2} \mathrm{H}_{2} \mathrm{~N}_{4} \mathrm{O}_{3}$ & $130.06 \mathrm{~g} / \mathrm{mol}$ \\
\hline 5 & 25.238 & 14.07 & Pyrazole-4-Carboxylic acid & $\mathrm{C}_{5} \mathrm{H}_{6} \mathrm{~N}_{2} \mathrm{O}_{2}$ & $126.11 \mathrm{~g} / \mathrm{mol}$ \\
\hline 6 & 25.892 & 8.49 & $2(3 \mathrm{H})$ Furanone & $\mathrm{C}_{4} \mathrm{H}_{4} \mathrm{O}_{2}$ & $84.07 \mathrm{~g} / \mathrm{mol}$ \\
\hline
\end{tabular}


Fig 1.1 GCMS reading of Aqueous extract of Adathodai Kudineer

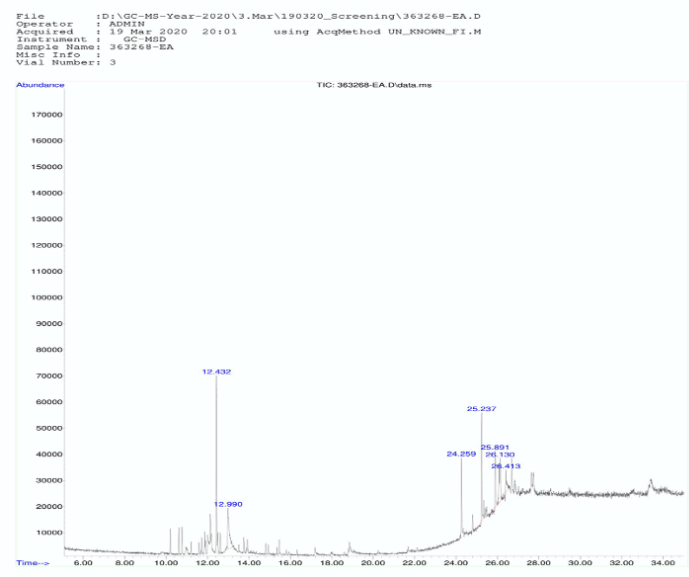

Fig 1.2 GCMS result of Ethanolic extract of Adathodaikudineer

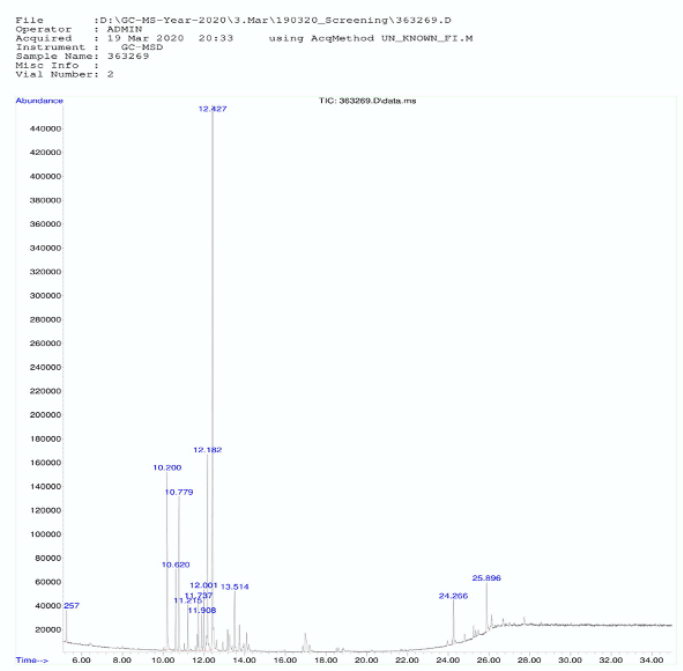

Table 1: Phytocomponents identified in the Ethanolic extract of Medicinal compound AdathodaiKudineer

\begin{tabular}{|c|c|c|}
\hline S.No & RT & Area \% \\
\hline 1 & 5.259 & 1.86 \\
\hline 2 & 5.259 & 1.86 \\
\hline 3 & 10.200 & 11.06 \\
\hline 4 & 10.622 & 3.73 \\
\hline 5 & 10.777 & 7.84 \\
\hline 6 & 11.734 & 3.32 \\
\hline 7 & 11.907 & 1.89 \\
\hline 8 & 12.002 & 3.36 \\
\hline 9 & 12.424 & 43.10 \\
\hline 10 & 13.512 & 3.07 \\
\hline 1 & 25.898 & 3.65 \\
\hline
\end{tabular}
Library/ID
(C: :DatabaselNIST11.L)
Eucalyptol
Toluene
2-Carene
Alpha-Copaene
1,6-Cyclodecadiene
Aromadendrene
Gamma-Murolene
Beta-Copaene
Cubebol
Selina-3,7 (11)-Diene
2-Butanone

Mol. F

$\mathrm{C}_{10} \mathrm{H}_{18} \mathrm{O}$

$\mathrm{C}_{7} \mathrm{H}_{8}$

$\mathrm{C}_{10} \mathrm{H}_{16}$

$\mathrm{C}_{15} \mathrm{H}_{24}$

$\mathrm{C}_{10} \mathrm{H}_{16}$

$\mathrm{C}_{15} \mathrm{H}_{24}$

$\mathrm{C}_{15} \mathrm{H}_{24}$

$\mathrm{C}_{15} \mathrm{H}_{24}$

$\mathrm{C}_{15} \mathrm{H}_{26} \mathrm{O}$

$\mathrm{C}_{15} \mathrm{H}_{24}$

$\mathrm{C}_{4} \mathrm{H}_{8} \mathrm{O}$
Mol. W (g/mol)

$154.25 \mathrm{~g} / \mathrm{mol}$

$92.14 \mathrm{~g} / \mathrm{mol}$

$136.23 \mathrm{~g} / \mathrm{mol}$

$204.35 \mathrm{~g} / \mathrm{mol}$

$136.23 \mathrm{~g} / \mathrm{mol}$

$204.35 \mathrm{~g} / \mathrm{mol}$

$204.35 \mathrm{~g} / \mathrm{mol}$

$204.35 \mathrm{~g} / \mathrm{mol}$

$222.37 \mathrm{~g} / \mathrm{mol}$

$204.35 \mathrm{~g} / \mathrm{mol}$

$72.11 \mathrm{~g} / \mathrm{mol}$

Molecular docking was performed between compounds identified in Siddha medicine Adathodai Kudineer with targets SARS-CoV-2 Virus main protease and Spike protein bound with ACE2 receptor, the results of the binding affinity of these compound with their respective targets are given in (Table 2).

Table 2: Binding affinity of the compounds with their respective targets

\begin{tabular}{|c|l|c|c|c|}
\hline S.no & Compound name & PubChem ID & $\begin{array}{c}\text { Binding energy for } \\
\text { PDBID:6LU7 } \\
\text { (KCal/mol) }\end{array}$ & $\begin{array}{c}\text { Binding energyfor } \\
\text { PDBID:6LZG } \\
\text { (Kcal/mol) }\end{array}$ \\
\hline 1 & Naphthalene & 931 & -5.5 & -6.3 \\
\hline 2 & Benzene Propanol & 31234 & -5.0 & -6.9 \\
\hline 3 & Benzene Acetic Acid & 327550 & -5.9 & -6.9 \\
\hline 4 & Furazan-3-Amine & 543119 & -4.6 & -5.2 \\
\hline 5 & Pyrazol-4-Carboxylic acid & 643160 & -4.5 & -5.1 \\
\hline 6 & $2(3 \mathrm{H})$ Furanone & 140765 & -3.6 & -4.1 \\
\hline 7 & Eucalyptol & 2758 & -5.1 & -5.2 \\
\hline 8 & Toluene & 1140 & -4.2 & -5.4 \\
\hline 9 & 2-Carene & 78249 & -4.9 & -6.3 \\
\hline 10 & Copaene & 19725 & -5.9 & -6.5 \\
\hline 11 & Alpha-Copaene & 70678558 & -8.2 & -10.9 \\
\hline 12 & 1,6-Cyclodecadiene & 5365639 & -5.2 & -5.6 \\
\hline 13 & Allomadendrene & 42608158 & -5.4 & -6.5 \\
\hline 14 & Aromadendrene & 91354 & -6.5 & -7.0 \\
\hline 15 & Gamma-Muurolene & 12313020 & -5.9 & -8.0 \\
\hline 16 & Beta-Copaene & 87529 & -5.8 & -6.6 \\
\hline 17 & Cubebol & 11276107 & -6.6 & -7.4 \\
\hline 18 & Selina-3,7 (11)-Diene & 522296 & -6.1 & -7.8 \\
\hline 19 & 2-Butanone & 6569 & 3.1 & -3.8 \\
\hline
\end{tabular}




\begin{tabular}{|c|c|c|c|}
\hline $\begin{array}{l}\text { S. } \\
\text { no }\end{array}$ & $\begin{array}{l}\text { Compound } \\
\text { name }\end{array}$ & $\begin{array}{l}\text { 3D Docking } \\
\text { pose of } \\
\text { compounds } \\
\text { with target } \\
6 \mathrm{LU} 7\end{array}$ & $\begin{array}{l}\text { 3D Docking } \\
\text { pose of } \\
\text { compounds } \\
\text { with target } \\
6 \mathrm{LZG}\end{array}$ \\
\hline 1 & Naphthalene & & \\
\hline 2 & $\begin{array}{l}\text { Benzene } \\
\text { Propanol }\end{array}$ & & \\
\hline 3 & $\begin{array}{c}\text { Benzene } \\
\text { Acetic Acid }\end{array}$ & & \\
\hline 4 & $\begin{array}{c}\text { Furazan-3- } \\
\text { Amine }\end{array}$ & & \\
\hline 5 & $\begin{array}{l}\text { Pyrazol-4- } \\
\text { Carboxylic } \\
\text { acid }\end{array}$ & & \\
\hline 6 & $\begin{array}{c}2(3 \mathrm{H}) \\
\text { Furanone }\end{array}$ & & \\
\hline 7 & Eucalyptol & & \\
\hline 8 & Toluene & & \\
\hline 9 & 2-Carene & & \\
\hline 10 & Copaene & & \\
\hline 11 & $\begin{array}{l}\text { Alpha- } \\
\text { Copaene }\end{array}$ & & \\
\hline 12 & $\begin{array}{c}1,6- \\
\text { Cyclodecadie } \\
\text { ne }\end{array}$ & & \\
\hline 13 & $\begin{array}{l}\text { Allomadendre } \\
\text { ne }\end{array}$ & & \\
\hline 14 & $\begin{array}{c}\text { Aromadendre } \\
\text { ne }\end{array}$ & & \\
\hline 15 & $\begin{array}{l}\text { Gamma- } \\
\text { Muurolene }\end{array}$ & & \\
\hline 16 & Beta-Copaene & & \\
\hline 17 & Cubebol & & \\
\hline 18 & $\begin{array}{l}\text { Selina-3,7 } \\
\text { (11)-Diene }\end{array}$ & & \\
\hline 19 & 2-Butanone & & \\
\hline
\end{tabular}

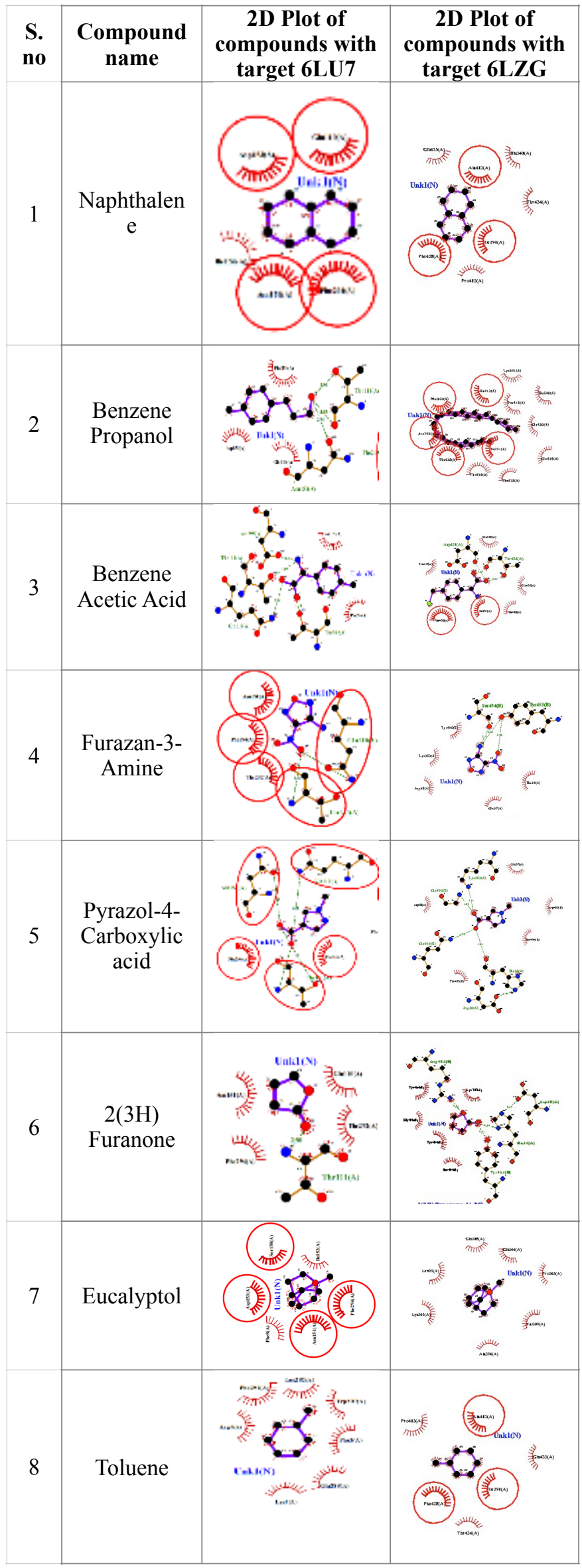




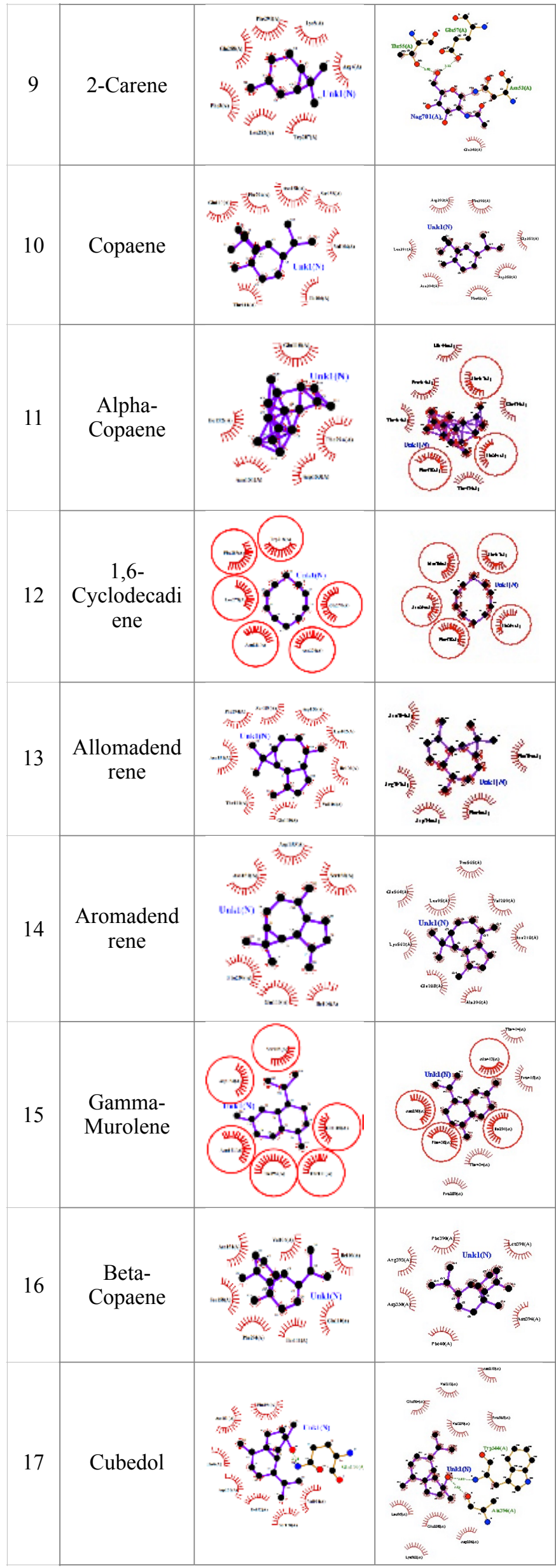

18

19

2-Butanone

Selina-3,7

(11)-Diene
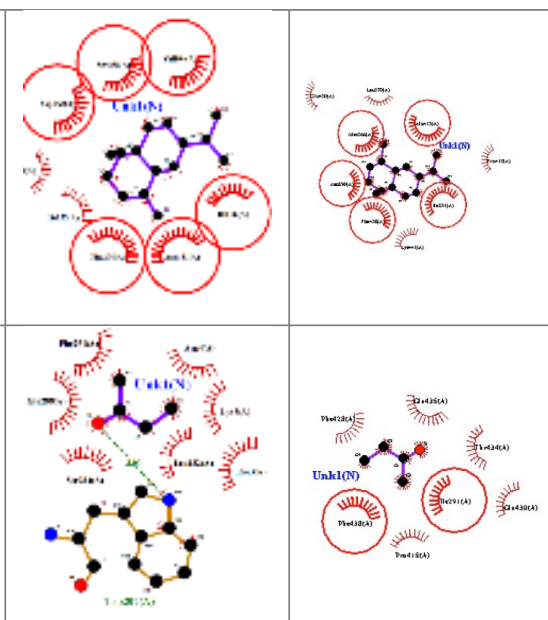

\section{Discussion}

ACE2 receptors are used by SARS-CoV-2 in the entry of the target cells to cause Corona Viral Disease 19 in Humans (2). The above mentioned compounds present in the aqueous and ethanolic extract of Adathodai kudineer Such as Napthalene, Benzene Propanol, Benzene Acetic Acid, Furazan-3-amine, Pyrazol-4-Carboxylic acid, 2(3H) Furanone, Eucalyptol, Toluene, 2-Carene, Alpha-Copaene, 1,6-Cyclodecadiene, Aromadendrene, Gamma-muurolene, Beta-copaene, Cubebol, Selina-3,7 (11) - Diene, 2Butanonehaving minimum binding affinity against Spike protein (PDB ID 6LU7) and Spike protein ACE2 complex domain (PDB ID 6LZG) based on the molecular docking results. This indicates that there is a significant possibility of this molecules to inhibit the viral entry into target cellular pathway and replication. The exact mechanism behind this have to be studied further in various dimensions.

Molecular docking binding energy results and case report analysis on Justicia adhatoda L. leaves extract use for COVID 19 strongly recommended by researchers in prevention and treatment aspects (12)(13)(14). So, the therapeutic potency of this herbal present in AK will give a major impact for the treatment of SARS COV 2 infection. Research study confirms that the alcoholic extract of Taxus baccata has bronchodilator effect and can be able to decrease the bronchial hyper reactivity in experimental animals (15) The compound Glycyrrhizin present in the plant Glycyrrhiza glabra $L$. has antiviral effect. This plant has potent antiviral effect against Cytomegalo virus, SARS virus, Hepatitis A, B, $C$ [16]. The Presence of Piperamides in the plant Piper Longum $L$. possess anti-viral against viruses related to upper respiratory tract infections and anti-proliferative effect (17).

Figure 3: ADME Alpha Copaene

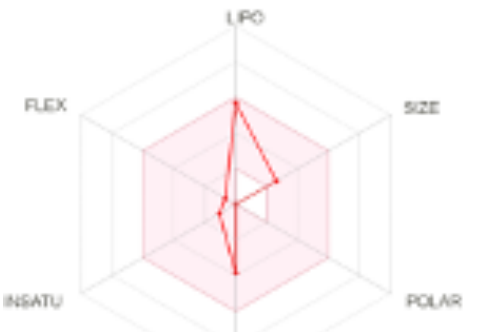




\section{Conclusion}

The lead biomolecules which present in the Siddha drug Adathodai Kudineer such as AlphaCopaene, Gamma-Muurolene, Selina-3,7 (11) - Diene, Cubebol, Aromadendrene have higher binding affinity towards theSARS-CoV-2 Spike Protein and ACE2 Receptor Spike protein Complex. Based on the findings of docking score values we can strongly suggest this Siddha poly herbal formulation Adathodai kudineer for the better management of COVID 19. Further preclinical and clinical trials have to be conducted in order to know the exact mechanism and efficacy of Adathodai Kudineer in SARS-CoV2 infection management.

\section{References}

1. https://covid19.who.int/

2. Astuti I, Ysrafil. Severe Acute Respiratory Syndrome Coronavirus 2 (SARS-CoV-2): An overview of viral structure and host response. Diabetes Metab Syndr Clin Res Rev 2020;14:40712. https://doi.org/10.1016/j.dsx.2020.04.020.

3. Christian GJ, Subramanian M, Periyasami D, Manickavasakam K, Gunasekaran P, Sivasubramanian S, et al. PROTECTIVE EFFECT OF POLYHERBAL SIDDHA FORMULATIONNILAVEMBU KUDINEER AGAINST COMMON VIRAL FEVERS INCLUDING DENGUE-A CASE-CONTROL APPROACH. Int J Pharm Sci Res 2015;6:1656-60. https://doi.org/10.13040/ IJPSR.0975-8232.6(4).1656-60.

4. arasi K, Gladys J. A COMBINATION OF Nilavembu Kudineer AND Adathodai Manapagu IN THE MANAGEMENT OF DENGUE FEVER, International Journal of Current Research Vol. 5, Issue, 04, pp.978-981, April, 2013.

5. Kiran G, Karthik L, Shree Devi MS, Sathiyarajeswaran P, Kanakavalli K, Kumar KM, et al. In Silico computational screening of Kabasura Kudineer - Official Siddha Formulation and JACOM against SARS-CoV-2 spike protein. J Ayurveda Integr Med 2020. https://doi.org/10.1016/ j.jaim.2020.05.009.

6. Kullappan Shanmugam U, Elango V, Natarajan K, Thiyagarajan B. STANDARDIZATION OF SIDDHA FORMULATION-ADHATODAI KUDINEER. International Journal of Developmental Research, Vol. 07, Issue, 10, pp.16077-16082, October, 2017

7. Kuppusamy mudaliar. K. N. Uthamarayan K. S. Siddha vaithya thirattu; edition : 2014; Department of Indian medicine and homeopathy - Chennai 106; pg. no. 259

8. Murugesan Mudaliar KS, Gunapadam porut panbu nool - part $-1 ; 7^{\text {th }}$ ed. Chennai; Department of Indian Medicine and Homeopathy; 2003.

9. Ihlenfeldt WD. PubChem. In: Applied Chemoinformatics, 2018, 245-258. [https://doi.org/ 10.1002/9783527806539.ch6e].

10. Wallace AC, Laskowski RA, Thornton JM. LIGPLOT: a program to generate schematic diagrams of protein-ligand interactions The LIGPLOT program automatically generates schematic 2-D representations of protein-ligand complexes from standard Protein Data Bank file input. vol. 8. 1995.

11. Daina A, Michielin O, Zoete V. SwissADME: A free web tool to evaluate pharmacokinetics, druglikeness and medicinal chemistry friendliness of small molecules. Sci Rep 2017;7. https://doi.org/ $10.1038 /$ srep42717.

12. Pugazhenthan Thangaraju, Sree Sudha Ty, Praveen Kumar Pasala, Sri Hari Ty, Sajitha Venkatesan, Eswaran Thangaraju ; The Role of Justicia Adhatoda as prophylaxis for COVID-19 Analysis based on docking study; 2021 Jan 19 ; Infect Disord Drug Targets; doi: $10.2174 / 1871526521666210119120643$. Online ahead of print.

13. Chavan R, Chowdhary A, In vitro inhibitory activity of Justicia adhatoda extracts against influenza virus infection and hemagglutination. Int. J. Pharm. Sci. Rev. Res. 2014;25(2):231-6

14. Singh B, Sharma RA.; Anti-inflammatory and antimicrobial properties of pyrroloquinazoline alkaloids from Adhatoda vasica Nees; Phytomedicine; Pubmed; 2013 Mar 15;20(5):441-5.

15. PK Patel, KV Patel, TR Gandhi, Evaluation of Effect of Taxus baccata Leaves Extract on Bronchoconstriction and Bronchial Hyperreactivity in Experimental Animals, J Young Pharm. 2011 Jan-Mar; 3(1): 41-47

16. Fiore C, Eisenhut M, Krausse R, Ragazzi E, Pellati D, Armanini D, et al. ANTIVIRAL EFFECTS OF GLYCYRRHIZA SPECIES 141 Antiviral Effects of Glycyrrhiza species. Phytother Res 2008;22:141-8. https://doi.org/10.1002/ptr.

17. CE Mair, R Liu, AG Atanasov, M Schmidtke, VM Dirsch, JM Rollinger, Antiviral and antiproliferative in vitro activities of piperamides from black pepper, Planta Med 2016; 82(S 01): S1-S381. 


\section{Appendix}

ADME properties of all active compounds of AdathodaiKudineer

Table 3: Physiochemical properties of active compounds of Adathodaikudineer formulation predicted from SwissADME

\begin{tabular}{|c|c|c|c|c|c|c|c|c|c|c|c|}
\hline $\begin{array}{l}\text { Sl. } \\
\text { No }\end{array}$ & Molecule & Formula & MW & $\begin{array}{c}\text { \#Heavy } \\
\text { atoms }\end{array}$ & $\begin{array}{c}\text { \#Aromatic } \\
\text { heavy atoms }\end{array}$ & $\begin{array}{c}\text { Fraction } \\
\text { Csp3 }\end{array}$ & $\begin{array}{c}\text { \#Rotata } \\
\text { ble } \\
\text { bonds }\end{array}$ & $\begin{array}{l}\text { \#H-bond } \\
\text { acceptors }\end{array}$ & $\begin{array}{c}\text { \#H-bond } \\
\text { donors }\end{array}$ & MR & TPSA \\
\hline 1 & Naphthalene & $\mathrm{C} 10 \mathrm{H} 8$ & 128.17 & 10 & 10 & 0 & 0 & 0 & 0 & 43.95 & 0 \\
\hline 2 & $\begin{array}{l}\text { Benzene } \\
\text { Propanol }\end{array}$ & $\mathrm{C} 9 \mathrm{H} 12 \mathrm{O}$ & 136.19 & 10 & 6 & 0.33 & 3 & 1 & 1 & 42.18 & 20.23 \\
\hline 3 & $\begin{array}{c}\text { Benzene Acetic } \\
\text { Acid }\end{array}$ & $\begin{array}{c}\mathrm{C} 9 \mathrm{H} 10 \mathrm{BrNO} \\
2\end{array}$ & 244.09 & 13 & 6 & 0.22 & 3 & 3 & 2 & 53.53 & 63.32 \\
\hline 4 & $\begin{array}{c}\text { Furazan-3- } \\
\text { Amine }\end{array}$ & $\mathrm{C} 2 \mathrm{H} 2 \mathrm{~N} 4 \mathrm{O} 3$ & 130.06 & 9 & 5 & 0 & 1 & 5 & 1 & 27.52 & 110.76 \\
\hline 5 & $\begin{array}{c}\text { Pyrazol-4- } \\
\text { Carboxylic acid }\end{array}$ & $\mathrm{C} 5 \mathrm{H} 6 \mathrm{~N} 2 \mathrm{O} 2$ & 126.11 & 9 & 5 & 0.2 & 1 & 3 & 1 & 30.45 & 55.12 \\
\hline 6 & $2(3 \mathrm{H})$ Furanone & $\mathrm{C} 4 \mathrm{H} 4 \mathrm{O} 2$ & 84.07 & 6 & 0 & 0.25 & 0 & 2 & 0 & 20.04 & 26.3 \\
\hline 7 & Eucalyptol & $\mathrm{C} 10 \mathrm{H} 18 \mathrm{O}$ & 154.25 & 11 & 0 & 1 & 0 & 1 & 0 & 47.12 & 9.23 \\
\hline 8 & Toluene & C7H8 & 92.14 & 7 & 6 & 0.14 & 0 & 0 & 0 & 31.41 & 0 \\
\hline 9 & 2-Carene & $\mathrm{C} 10 \mathrm{H} 16$ & 136.23 & 10 & 0 & 0.8 & 0 & 0 & 0 & 45.22 & 0 \\
\hline 10 & Copaene & $\mathrm{C} 15 \mathrm{H} 24$ & 204.35 & 15 & 0 & 0.87 & 1 & 0 & 0 & 67.14 & 0 \\
\hline 11 & Alpha-Copaene & $\mathrm{C} 15 \mathrm{H} 24$ & 204.35 & 15 & 0 & 0.87 & 1 & 0 & 0 & 67.14 & 0 \\
\hline 12 & $\begin{array}{c}1,6- \\
\text { Cyclodecadiene }\end{array}$ & $\mathrm{C} 10 \mathrm{H} 16$ & 136.23 & 10 & 0 & 0.6 & 0 & 0 & 0 & 47.12 & 0 \\
\hline 13 & Allomadendrene & $\mathrm{C} 15 \mathrm{H} 24$ & 204.35 & 15 & 0 & 0.87 & 0 & 0 & 0 & 67.14 & 0 \\
\hline 14 & Aromadendrene & $\mathrm{C} 15 \mathrm{H} 24$ & 204.35 & 15 & 0 & 0.87 & 0 & 0 & 0 & 67.14 & 0 \\
\hline 15 & $\begin{array}{l}\text { Gamma- } \\
\text { Muurolene }\end{array}$ & $\mathrm{C} 15 \mathrm{H} 24$ & 204.35 & 15 & 0 & 0.73 & 1 & 0 & 0 & 69.04 & 0 \\
\hline 16 & Beta-Copaene & $\mathrm{C} 15 \mathrm{H} 24$ & 204.35 & 15 & 0 & 0.87 & 1 & 0 & 0 & 67.14 & 0 \\
\hline 17 & Cubedol & $\mathrm{C} 15 \mathrm{H} 26 \mathrm{O}$ & 222.37 & 16 & 0 & 1 & 1 & 1 & 1 & 68.82 & 20.23 \\
\hline
\end{tabular}

Table 4: Liphophilicity of active compounds of Adathodaikudineer formulation predicted from SwissADME

\begin{tabular}{|c|c|c|c|c|c|c|c|}
\hline Sl.No & Molecule & iLOGP & XLOGP3 & WLOGP & MLOGP & Silicos-IT Log P & Consensus Log P \\
\hline 1 & Naphthalene & 1.99 & 3.3 & 2.84 & 4.26 & 3.11 & 3.1 \\
\hline 2 & Benzene Propanol & 1.95 & 1.88 & 1.61 & 2.19 & 2.35 & 2 \\
\hline 3 & Benzene Acetic Acid & 1.34 & -1.14 & 1.19 & -0.66 & 1.55 & 0.46 \\
\hline 4 & Furazan-3-Amine & 0.31 & 0.12 & -0.43 & -1.45 & -1.42 & -0.57 \\
\hline 5 & Pyrazol-4-Carboxylic acid & 0.81 & -0.24 & 0.12 & -0.4 & -0.26 & 0.01 \\
\hline 6 & 2(3H) Furanone & 1.19 & 0.22 & 0.45 & -0.01 & 1.02 & 0.57 \\
\hline 7 & Eucalyptol & 2.58 & 2.74 & 2.74 & 2.45 & 2.86 & 2.67 \\
\hline 8 & Toluene & 1.85 & 2 & 2.73 & 3.52 & 2.44 & 2.51 \\
\hline 9 & 2-Carene & 2.66 & 3 & 2.85 & 4.29 & 2.79 & 3.12 \\
\hline 10 & Copaene & 3.4 & 4.27 & 4.47 & 5.65 & 3.73 & 4.3 \\
\hline 11 & Alpha-Copaene & 3.4 & 4.27 & 4.47 & 5.65 & 3.73 & 4.3 \\
\hline 12 & 1,6-Cyclodecadiene & 2.57 & 3.45 & 3.84 & 3.27 & 2.73 & 3.17 \\
\hline 13 & Allomadendrene & 3.27 & 4.27 & 4.71 & 5.65 & 3.8 & 4.34 \\
\hline 14 & Aromadendrene & 3.27 & 4.27 & 4.71 & 5.65 & 3.8 & 4.34 \\
\hline 15 & Gamma-Muurolene & 3.38 & 4.58 & 4.31 & 4.63 & 4.01 & 4.18 \\
\hline 16 & Beta-Copaene & 3.35 & 4.27 & 4.71 & 5.65 & 4.01 & 4.4 \\
\hline 17 & Cubedol & 3.09 & 3.47 & 3.92 & 3.81 & 3.22 & 3.5 \\
\hline
\end{tabular}

Table 5: Water solubility properties of all the active compounds of AdathodaiKudineer predicted from SWISS ADME

\begin{tabular}{|c|c|c|c|c|c|c|c|c|c|c|c|}
\hline $\begin{array}{l}\text { ESOL } \\
\log S\end{array}$ & $\begin{array}{c}\text { ESOL } \\
\text { Solubili } \\
\text { ty (mg/ } \\
\text { ml) }\end{array}$ & $\begin{array}{c}\text { ESOL } \\
\text { Solubili } \\
\text { ty (mol/ } \\
\text { l) }\end{array}$ & ESOL Class & $\begin{array}{c}\text { Ali Log } \\
\text { S }\end{array}$ & $\begin{array}{c}\text { Ali } \\
\text { Solubili } \\
\text { ty (mg/ } \\
\text { ml) }\end{array}$ & $\begin{array}{c}\text { Ali } \\
\text { Solubili } \\
\text { ty (mol/ } \\
\text { l) }\end{array}$ & Ali Class & $\begin{array}{l}\text { Silicos- } \\
\text { IT } \\
\text { LogSw }\end{array}$ & $\begin{array}{c}\text { Silicos- } \\
\text { IT } \\
\text { Solubili } \\
\text { ty (mg/ } \\
\text { ml) }\end{array}$ & $\begin{array}{l}\text { Silicos- } \\
\text { IT } \\
\text { Solubili } \\
\text { ty (mol/ } \\
\text { l) }\end{array}$ & Silicos-IT class \\
\hline-3.45 & $4.51 \mathrm{E}-02$ & $3.52 \mathrm{E}-04$ & Soluble & -2.98 & $1.36 \mathrm{E}-01$ & $1.06 \mathrm{E}-03$ & Soluble & -4.03 & 1.19E-02 & $9.27 \mathrm{E}-05$ & $\begin{array}{c}\text { Moderately } \\
\text { soluble }\end{array}$ \\
\hline-2.11 & $1.05 \mathrm{E}+00$ & $7.68 \mathrm{E}-03$ & Soluble & -1.93 & $1.61 \mathrm{E}+00$ & $1.18 \mathrm{E}-02$ & Very soluble & -3 & $1.35 \mathrm{E}-01$ & 9.89E-04 & Soluble \\
\hline-0.78 & $4.06 \mathrm{E}+01$ & $1.66 \mathrm{E}-01$ & Very soluble & 0.3 & $4.89 \mathrm{E}+02$ & $2.00 \mathrm{E}+00$ & Highly soluble & -2.72 & $4.61 \mathrm{E}-01$ & $1.89 \mathrm{E}-03$ & Soluble \\
\hline-1.07 & $1.11 \mathrm{E}+01$ & $8.57 \mathrm{E}-02$ & Very soluble & -2 & $1.30 \mathrm{E}+00$ & $9.96 \mathrm{E}-03$ & Soluble & 0.15 & $1.83 \mathrm{E}+02$ & $1.41 \mathrm{E}+00$ & Soluble \\
\hline-0.82 & $1.93 \mathrm{E}+01$ & $1.53 \mathrm{E}-01$ & Very soluble & -0.46 & $4.38 \mathrm{E}+01$ & $3.47 \mathrm{E}-01$ & Very soluble & -0.13 & $9.37 \mathrm{E}+01$ & $7.43 \mathrm{E}-01$ & Soluble \\
\hline-0.5 & $2.66 \mathrm{E}+01$ & $3.16 \mathrm{E}-01$ & Very soluble & -0.33 & $3.92 \mathrm{E}+01$ & $4.66 \mathrm{E}-01$ & Very soluble & -0.04 & $7.67 \mathrm{E}+01$ & $9.12 \mathrm{E}-01$ & Soluble \\
\hline-2.52 & 4.63E-01 & $3.00 \mathrm{E}-03$ & Soluble & -2.59 & $3.98 \mathrm{E}-01$ & $2.58 \mathrm{E}-03$ & Soluble & -2.45 & $5.45 \mathrm{E}-01$ & $3.53 \mathrm{E}-03$ & Soluble \\
\hline
\end{tabular}


Christian G J et.al., Computational efficacy of active compounds in Adathodai Kudineer a classical Siddha herbal formulation

\begin{tabular}{|c|c|c|c|c|c|c|c|c|c|c|c|}
\hline-2.77 & $1.58 \mathrm{E}-01$ & $1.72 \mathrm{E}-03$ & Soluble & -2.38 & $3.80 \mathrm{E}-01$ & $4.13 \mathrm{E}-03$ & Soluble & -2.69 & $1.88 \mathrm{E}-01$ & $2.04 \mathrm{E}-03$ & Soluble \\
\hline-2.48 & $4.51 \mathrm{E}-01$ & $3.31 \mathrm{E}-03$ & Soluble & -2.51 & $4.22 \mathrm{E}-01$ & $3.10 \mathrm{E}-03$ & Soluble & -2.23 & $8.06 \mathrm{E}-01$ & $5.92 \mathrm{E}-03$ & Soluble \\
\hline-3.86 & $2.84 \mathrm{E}-02$ & $1.39 \mathrm{E}-04$ & Soluble & -4.19 & $1.32 \mathrm{E}-02$ & $6.46 \mathrm{E}-05$ & $\begin{array}{c}\text { Moderately } \\
\text { soluble }\end{array}$ & -3.07 & $1.74 \mathrm{E}-01$ & $8.51 \mathrm{E}-04$ & Soluble \\
\hline-3.86 & $2.84 \mathrm{E}-02$ & $1.39 \mathrm{E}-04$ & Soluble & -4.19 & $1.32 \mathrm{E}-02$ & $6.46 \mathrm{E}-05$ & $\begin{array}{c}\text { Moderately } \\
\text { soluble }\end{array}$ & -3.07 & $1.74 \mathrm{E}-01$ & $8.51 \mathrm{E}-04$ & Soluble \\
\hline-3.1 & $1.07 \mathrm{E}-01$ & 7.87E-04 & Soluble & -3.54 & $3.97 \mathrm{E}-02$ & $2.91 \mathrm{E}-04$ & Soluble & -1.7 & $2.75 \mathrm{E}+00$ & 2.02E-02 & Soluble \\
\hline-4.07 & $1.72 \mathrm{E}-02$ & $8.43 \mathrm{E}-05$ & $\begin{array}{l}\text { Moderately } \\
\text { soluble }\end{array}$ & -4.44 & 7.44E-03 & $3.64 \mathrm{E}-05$ & $\begin{array}{c}\text { Moderately } \\
\text { soluble }\end{array}$ & -3.32 & $9.83 \mathrm{E}-02$ & $4.81 \mathrm{E}-04$ & Soluble \\
\hline-4.07 & $1.72 \mathrm{E}-02$ & $8.43 \mathrm{E}-05$ & $\begin{array}{c}\text { Moderately } \\
\text { soluble }\end{array}$ & -4.44 & 7.44E-03 & $3.64 \mathrm{E}-05$ & $\begin{array}{c}\text { Moderately } \\
\text { soluble }\end{array}$ & -3.32 & $9.83 \mathrm{E}-02$ & $4.81 \mathrm{E}-04$ & Soluble \\
\hline-3.76 & $3.58 \mathrm{E}-02$ & $1.75 \mathrm{E}-04$ & Soluble & -4.02 & $1.94 \mathrm{E}-02$ & $9.47 \mathrm{E}-05$ & $\begin{array}{c}\text { Moderately } \\
\text { soluble }\end{array}$ & -3.32 & $9.83 \mathrm{E}-02$ & $4.81 \mathrm{E}-04$ & Soluble \\
\hline-4.01 & $2.00 \mathrm{E}-02$ & $9.81 \mathrm{E}-05$ & $\begin{array}{c}\text { Moderately } \\
\text { soluble }\end{array}$ & -4.44 & 7.44E-03 & $3.64 \mathrm{E}-05$ & $\begin{array}{c}\text { Moderately } \\
\text { soluble }\end{array}$ & -3.32 & 9.83E-02 & $4.81 \mathrm{E}-04$ & Soluble \\
\hline-3.62 & $5.31 \mathrm{E}-02$ & $2.39 \mathrm{E}-04$ & Soluble & -4.04 & $2.01 \mathrm{E}-02$ & $9.04 \mathrm{E}-05$ & $\begin{array}{c}\text { Moderately } \\
\text { soluble }\end{array}$ & -2.73 & 4.10E-01 & $1.85 \mathrm{E}-03$ & Soluble \\
\hline-3.9 & $2.55 \mathrm{E}-02$ & $1.25 \mathrm{E}-04$ & Soluble & -4.16 & $1.42 \mathrm{E}-02$ & $6.94 \mathrm{E}-05$ & $\begin{array}{l}\text { Moderately } \\
\text { soluble }\end{array}$ & -3.75 & 3.63E-02 & $1.78 \mathrm{E}-04$ & Soluble \\
\hline-0.4 & $2.85 \mathrm{E}+01$ & $3.95 \mathrm{E}-01$ & Very soluble & -0.21 & $4.44 \mathrm{E}+01$ & $6.16 \mathrm{E}-01$ & Very soluble & -0.87 & $9.77 \mathrm{E}+00$ & $1.36 \mathrm{E}-01$ & Soluble \\
\hline
\end{tabular}

Table 6: Pharmacokinetic properties of all the active compounds of AdathodaiKudineer predicted from SWISS

\begin{tabular}{|c|c|c|c|c|c|c|c|c|}
\hline \multicolumn{9}{|c|}{ ADME } \\
\hline $\begin{array}{c}\text { GI } \\
\text { absorption }\end{array}$ & $\begin{array}{c}\text { BBB } \\
\text { permeant }\end{array}$ & $\begin{array}{c}\text { Pgp } \\
\text { substrate }\end{array}$ & $\begin{array}{l}\text { CYP1A2 } \\
\text { inhibitor }\end{array}$ & $\begin{array}{c}\text { CYP2C19 } \\
\text { inhibitor }\end{array}$ & $\begin{array}{l}\text { CYP2C9 } \\
\text { inhibitor }\end{array}$ & $\begin{array}{l}\text { CYP2D6 } \\
\text { inhibitor }\end{array}$ & $\begin{array}{l}\text { CYP3A4 } \\
\text { inhibitor }\end{array}$ & $\underset{(\mathrm{cm} / \mathbf{s})}{\log \mathrm{Kp}}$ \\
\hline Low & Yes & No & Yes & No & No & No & No & -4.74 \\
\hline High & Yes & No & Yes & No & No & No & No & -5.8 \\
\hline High & Yes & No & No & No & No & No & No & -8.6 \\
\hline High & No & No & No & No & No & No & No & -7.01 \\
\hline High & No & No & No & No & No & No & No & -7.24 \\
\hline High & No & No & No & No & No & No & No & -6.66 \\
\hline High & Yes & No & No & No & No & No & No & -5.3 \\
\hline Low & No & No & Yes & No & No & No & No & -4.92 \\
\hline Low & Yes & No & No & No & No & No & No & -5.11 \\
\hline Low & Yes & No & Yes & Yes & Yes & No & No & -4.37 \\
\hline Low & Yes & No & Yes & Yes & Yes & No & No & -4.37 \\
\hline Low & Yes & No & No & No & No & No & No & -4.4 \\
\hline Low & Yes & No & Yes & Yes & Yes & No & No & -4.2 \\
\hline Low & Yes & No & Yes & Yes & Yes & No & No & -4.2 \\
\hline Low & No & No & No & Yes & Yes & No & No & -4.49 \\
\hline Low & Yes & No & Yes & Yes & Yes & No & No & -4.2 \\
\hline High & Yes & No & No & Yes & Yes & No & No & -4.87 \\
\hline Low & No & No & No & Yes & Yes & No & No & -4.39 \\
\hline High & Yes & No & No & No & No & No & No & -6.53 \\
\hline
\end{tabular}

Table 7: Drug likeliness of all the active compounds of AdathodaiKudineer predicted from SWISS ADME

\section{Lipinski} \#violations
Ghose \#violations Veber \#violations

\section{Egan \#violations}

\section{Muegge} \#violations
Bioavailability

Score

0.55

0.55

0.55

0.55

0.56

0.55

0.55

0.55

0.55

0.55

0.55

0.55

0.55

0.55

0.55 


\begin{tabular}{|l|l|l|l|l|l|}
\hline \hline 1 & 0 & 0 & 0 & 1 & 0.55 \\
\hline 0 & 0 & 0 & 0 & 1 & 0.55 \\
\hline 1 & 0 & 0 & 0 & 1 & 0.55 \\
\hline
\end{tabular}

Table 8: Medicinal chemistry properties of all the active compounds of AdathodaiKudineer predicted from SWISS ADME

\begin{tabular}{|c|c|c|c|}
\hline PAINS \#alerts & Brenk \#alerts & Leadlikeness \#violations & Synthetic Accessibility \\
\hline 0 & 0 & 1 & 1 \\
\hline 0 & 0 & 1 & 2.04 \\
\hline 0 & 1 & 1 & 2.8 \\
\hline 0 & 2 & 1 & 1.09 \\
\hline 0 & 0 & 1 & 2.33 \\
\hline 0 & 0 & 1 & 3.65 \\
\hline 0 & 0 & 1 & 1 \\
\hline 0 & 0 & 1 & 3.84 \\
\hline 0 & 1 & 1 & 4.62 \\
\hline 0 & 1 & 2 & 4.62 \\
\hline 0 & 1 & 2 & 2.99 \\
\hline 0 & 1 & 2 & 3.7 \\
\hline 0 & 1 & 2 & 3.7 \\
\hline 0 & 1 & 2 & 4.35 \\
\hline 0 & 1 & 2 & 3.91 \\
\hline
\end{tabular}

